\title{
Formação de Professores de Matemática: Avaliação de Recursos Digitais
}

\author{
Fernanda M. Santos ${ }^{1}$, Gilmara T. Barcelos ${ }^{1}$, Silvia Cristina F. Batista ${ }^{1}$ \\ ${ }^{1}$ Instituto Federal Fluminense Campus Campos-Centro \\ Rua Dr. Siqueira, 273. Parque Dom Bosco. CEP: 28030-130. Campos dos \\ Goytacazes/RJ \\ nanda.s.manhaes@gmail.com, gilmarabeiff.edu.br, silviac@iff.edu.br
}

\begin{abstract}
This article presents an analysis of a case study which aimed at contributing to the development of critical thinking of students of Mathematics Education regarding the selection and use of digital technologies as pedagogic resources. With this in mind, a mini course was offered in which students used an applet and related activities in a workbook. These resources are targeted to a topic in analytic geometry and were organized by the authors of this paper with software GeoGebra 5.0. In general, students manifested criticism in regard to the resources as they gave positive reviews as well as suggestions on how to improve them.
\end{abstract}

Resumo. Este artigo apresenta a análise de um estudo de caso com o qual se buscou contribuir para o desenvolvimento do senso crítico de licenciandos em Matemática, em relação à seleção e ao uso de tecnologias digitais como recursos pedagógicos. Nesse sentido, foi realizado um minicurso no qual foram utilizados um applet e uma apostila de atividades relacionadas ao mesmo. Tais recursos são direcionados a um tema de geometria analítica e foram elaborados pelas autoras deste artigo, por meio do software GeoGebra 5.0. Em geral, os licenciandos assumiram posturas críticas em relação aos recursos utilizados, avaliando-os positivamente, mas também propondo sugestões de melhorias.

\section{Introdução}

As tecnologias digitais (TD) favorecem a realização de experimentações, colaboram em atividades de investigação, facilitam visualizações, manipulações e levantamento de hipóteses. É fundamental, portanto, que essas tecnologias sejam utilizadas na educação formal, embora, certamente, aprendizagens continuem ocorrendo mesmo quando estas não são consideradas [Klopfer et al. 2009].

Em particular, na Matemática, a interatividade possibilitada pelas TD concede a alternativa de realizar experimentos e criar situações que potencializam o desenvolvimento do pensamento lógico [Zotto et al. 2013]. Borba (2011) menciona que as possibilidades de investigação e de experimentação propiciadas por essas tecnologias podem levar estudantes a estabelecer e testar conjecturas, levantando, assim, subsídios para a elaboração de uma demonstração matemática.

É importante ressaltar que o uso pedagógico das TD está diretamente relacionado às concepções pedagógicas dos professores. Dessa forma, é essencial que 
ocorram iniciativas direcionadas à formação inicial e continuada desses profissionais, tendo em vista a integração de recursos digitais à educação formal. Nesse contexto, se insere o presente trabalho, defendendo a importância da preparação de licenciandos para a avaliação de TD educacionais. A referida importância tem sido focalizada por vários pesquisadores [Borges 2008; Guedes e Castro-Filho 2010; Lima et al. 2012].

Tendo em vista contribuir para o desenvolvimento do senso crítico de licenciandos em Matemática, em relação à seleção e ao uso de TD como recursos pedagógicos, promoveu-se um estudo de caso. Neste, foram avaliados um applet ${ }^{1}$ e uma apostila de atividades relacionadas ao mesmo. Tais recursos foram desenvolvidos pelas autoras deste artigo visando apoiar o estudo dos casos particulares da equação geral do plano (tópico de geometria analítica). O referido applet foi elaborado por meio do software GeoGebra 5.0.

O GeoGebra é um software livre que permite o estudo de Geometria, Álgebra e Cálculo, além de apresentar características de um CAS (Computer Algebra System). Este pode ser utilizado em todos os níveis de ensino [Hohenwarter; Preiner 2007]. Atualmente, encontra-se disponível a versão $5.0^{2}$, que possui, além da maioria dos recursos habituais das versões anteriores, uma janela que permite fazer construções tridimensionais (janela 3D).

Para o estudo de caso foi organizado um questionário que visou captar a percepção dos participantes sobre os recursos desenvolvidos (applet e atividades) e sobre as possíveis contribuições dos mesmos para o estudo do tema focalizado. Os dados foram analisados segundo uma abordagem, predominantemente, qualitativa.

Diante desse panorama, o objetivo do presente artigo é descrever o estudo de caso e analisar os resultados obtidos no mesmo, tendo em vista incentivar ações destinadas à preparação de licenciandos para a avaliação do uso pedagógico de TD. Para tanto, na seção 2 , são promovidas reflexões sobre a importância da formação inicial de professores para a avaliação de TD e, na seção 3, são descritos os procedimentos metodológicos adotados no estudo de caso. Na seção 4, apresenta-se uma breve caracterização do software GeoGebra 5.0 e dos recursos desenvolvidos (applet e atividades) e, na seção 5, são discutidos os resultados obtidos. Finalizando, na seção 6, são apresentadas considerações sobre o estudo promovido.

\section{Formação Inicial de Professores e Avaliação de Tecnologias Digitais}

As TD e as mídias sociais têm contribuído para mudanças na vida cotidiana das pessoas, transformando as formas de entretenimento e de aprendizagem, entre outros aspectos [Nascimento 2013]. O momento e a forma como os professores adotam tecnologias são aspectos que influenciam, diretamente, na ocorrência, ou não, de melhorias nesse processo. Portanto, os professores são pontos chave do sistema educacional. O benefício alcançado com o uso de TD em sala de aula depende, entre outros aspectos, do preparo desses profissionais [Nascimento 2013].

Assim, considera-se de suma importância que ocorram iniciativas que contemplem a formação inicial e continuada de professores para a integração das TD ao

\footnotetext{
${ }^{1}$ Applets são programas desenvolvidos em linguagem de programação Java ${ }^{\circledR}$, que podem ser incluídos em códigos HTML [Deitel, H.; Deitel, P. 2003].

${ }^{2}$ Essa versão encontra-se disponível em <http://download.geogebra.org/installers/5.0/> e está em fase de testes para identificação de problemas.
} 
contexto escolar. Uma perspectiva teórica que pode fundamentar essas iniciativas é o Technological Pedagogical Content Knowledge (TPACK), ou seja, conhecimento tecnológico, pedagógico e do conteúdo. Define-se TPACK como o conhecimento que os professores necessitam ter para ensinar com e sobre tecnologias nas diversas áreas do conhecimento, incluindo a discussão de questões pedagógicas sobre o uso das TD no estudo de conteúdos [Mishra; Koehler 2006]. Destaca-se que, segundo esse referencial, o conhecimento tecnológico compreende, entre outros aspectos, a habilidade de aprender e de adaptar-se a uma nova tecnologia e a capacidade de selecionar e de utilizar diversos recursos [Mishra; Koehler 2006]. Defendendo a necessidade de um desenvolvimento profissional que inclua uma fundamentação teórica baseada em TPACK, Drijvers (2012) afirma que a integração das TD na educação formal não reduz a importância do professor. Este, na verdade, passa a ter outras funções, como a de estruturar as situações de aprendizagem com o uso dessas tecnologias.

Nesse contexto, a seleção de recursos é de fundamental importância e requer desenvolvimento de senso crítico e conhecimento de fatores a serem avaliados. Como defendido por Tarouco (2004), avaliações de objetos de aprendizagem digitais podem orientar um melhor uso dos mesmos e fornecer um feedback para os desenvolvedores, a fim de que os recursos sejam aprimorados. Batista (2004) afirma que, na educação escolar, as TD podem ser importantes recursos didáticos, porém, isso implica a necessidade de uma postura crítica do professor na seleção das mesmas. Tal postura pode contribuir para a identificação de ferramentas mais adequadas e melhor aproveitamento das potencialidades destas [Batista 2004].

Borges (2008) destaca a importância de preparar os professores para uma leitura crítica dos recursos tecnológicos e defende, ainda, a participação dos gestores no processo de formação, sensibilização e conscientização sobre o papel da tecnologia na educação. Segundo o referido autor, se o gestor não incentivar, o professor não utilizará tais recursos. Para Lima et al. (2012), é imprescindível analisar criticamente o uso das TD e sua influência na escola. Além disso, esses autores ressaltam que a tecnologia pode ser um produto construído por professores e alunos. Guedes e Castro-Filho (2010) defendem a importância de que professores adquiram condições de avaliar não só a qualidade de recursos tecnológicos, mas também, a finalidade de uso e as condições para a realização de ações com os mesmos.

Diante desse panorama, foi promovida a pesquisa relatada neste trabalho, cujos procedimentos metodológicos são descritos na seção seguinte.

\section{Estudo de Caso: Contexto e Procedimentos Metodológicos}

A partir do objetivo da pesquisa, optou-se por realizar um estudo de caso. Este foi promovido durante o minicurso denominado Investigando os Casos Particulares da Equação Geral do Plano: GeoGebra Janela 3D, realizado em um Instituto Federal de Educação, em maio de 2014, com duração de quatro horas.

Foram convidados todos os alunos do $5^{\circ}$ e do $7^{\circ}$ período da licenciatura em Matemática $^{3}$ da Instituição Federal considerada, totalizando 19 alunos. Destes, nove aceitaram o convite, mas apenas sete participaram, todos alunos do $7^{\circ}$ período. Optou-se por este público pelo fato do tema a ser abordado no minicurso clamar por requisitos mínimos que estes licenciandos possuíam. Esta informação já era conhecida pois, uma

\footnotetext{
${ }^{3} \mathrm{O}$ referido curso possui sete períodos e, como a entrada é anual, no primeiro semestre não há $6^{\circ}$ período.
} 
das autoras deste artigo é aluna do $7^{\circ}$ período da licenciatura em questão e outra é professora do referido curso.

A escolha do tema matemático casos particulares da equação geral do plano decorreu do fato do uso da janela 3D do GeoGebra possibilitar visualizações, por meio da movimentação dos planos, de forma simples, rápida e motivadora. A partir da definição do tema matemático, foi desenvolvido o applet Equação Geral do Plano e uma apostila de atividades para o estudo do mesmo. O applet foi disponibilizado ${ }^{4}$ na plataforma de compartilhamento GeoGebraTube ${ }^{5}$. Apresenta-se, na seção 4, uma descrição mais detalhada desse recurso e da apostila.

Antecedendo o uso do applet e a resolução das atividades, a janela 3D do GeoGebra 5.0 foi apresentada e alguns recursos foram experimentados. Os licenciandos conheciam apenas as ferramentas da janela 2D. Dando continuidade ao minicurso, o GeoGebraTube foi apresentado e o applet foi salvo (arquivo ggb) no computador que cada licenciando estava utilizando. A seguir, foi distribuída a apostila de atividades e, finalizando o estudo, promoveu-se uma discussão sobre o applet e as respostas das atividades.

Para a coleta de dados, foram adotados os seguintes instrumentos: observação, questionário e respostas das atividades. Os dados foram analisados segundo uma abordagem, predominantemente, qualitativa. Ações e comentários feitos durante o minicurso foram registrados para posterior análise.

Finalizando o minicurso, os licenciandos responderam a um questionário, disponível no Google Drive. Este continha perguntas abertas e fechadas, objetivando coletar dados que permitissem traçar um breve perfil dos participantes e analisar a visão dos mesmos sobre o applet e as atividades propostas. Além disso, buscou-se investigar como os licenciandos, como futuros professores, avaliavam o uso de applets, semelhantes ao utilizado, para abordagem de temas matemáticos. Finalizando, questionaram-se os pontos positivos e negativos do minicurso. Neste artigo, apresentase a análise dos dados considerados mais pertinentes ao relato.

\section{Descrição dos Recursos: GeoGebra, Applet e Apostila de Atividades}

O software GeoGebra 5.0 é livre e multiplataforma. Ao abrir o mesmo, a janela 3D não se encontra visível. Para visualizá-la, é preciso clicar em exibir, no menu principal e, em seguida, em Janela de Visualização 3D.

Clicando, com o botão direito do mouse, na janela de visualização 3D, tem-se a opção de retirar os eixos, a malha e o plano representado. Ao clicar, abre-se uma caixa (Figura 1) na qual são selecionados os elementos a serem retirados (estes não mais serão exibidos, mas é possível reverter isso, posteriormente, clicando, novamente, com o botão direito do mouse, e selecionando o item a ser exibido).

No GeoGebra o processo de elaboração de applets $^{6}$ é muito simples. Nas versões anteriores a 5.0 é possível gerá-los (arquivo HTML), salvá-los no próprio computador e, posteriormente, disponibilizá-los em sites, blogs e ambientes de aprendizagens. Para

\footnotetext{
${ }_{5}^{4}$ Disponível em: <http://www.geogebratube.org/material/show/id/113179>.

Ambiente para compartilhamento de recursos produzidos com o GeoGebra $<$ http://www.geogebratube.org/>.

${ }^{6}$ No GeoGebra, esses recursos são denominados planilhas dinâmicas.
} 
tanto, basta clicar em arquivo e, a seguir, em exportar planilha dinâmica como página web (HTML). Já na versão 5.0, as construções somente podem ser transformadas em applets direto no GeoGebraTube, por meio de upload, ou seja, não é gerado o arquivo HTML no computador. O caminho é o mesmo, porém é necessário ter uma conta no GeoGebraTube. A disponibilização nessa plataforma permite a pesquisa por materiais, a avaliação por usuários cadastrados e o envio de comentários [Hohenwarter 2013].

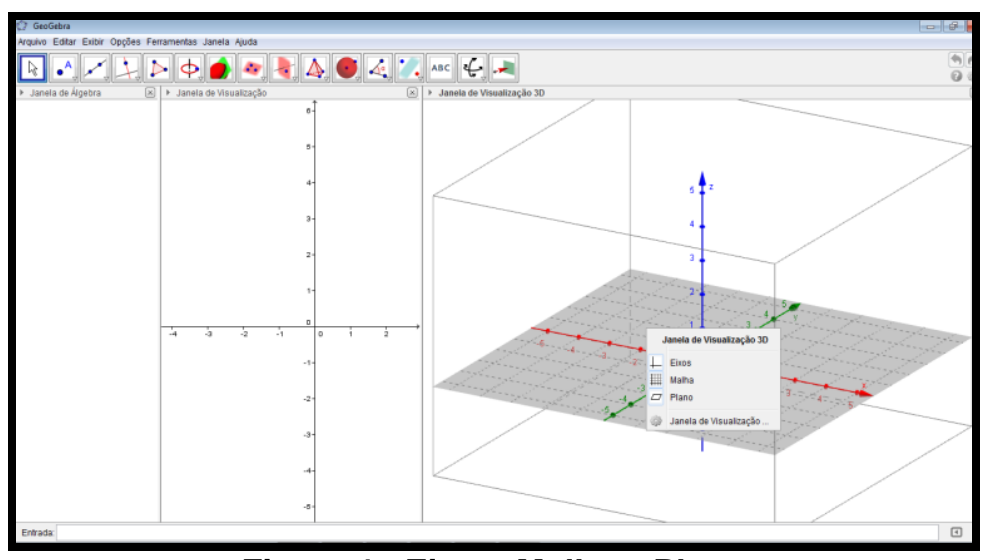

Figura 1. Eixos, Malha e Plano

Fonte: Tela capturada do software GeoGebra 5.0

O applet Equação Geral do Plano (Figura 2) contém quatro seletores $a, b, c$ e $d$ que correspondem, respectivamente, aos coeficientes e ao termo independente da equação geral do plano. Estes seletores variam de -20 a 20 , com incremento de 1 unidade. Assim, ao clicar e deslizar o mouse sobre os seletores, os coeficientes da equação geral do plano estarão variando e o plano mudando de posição.

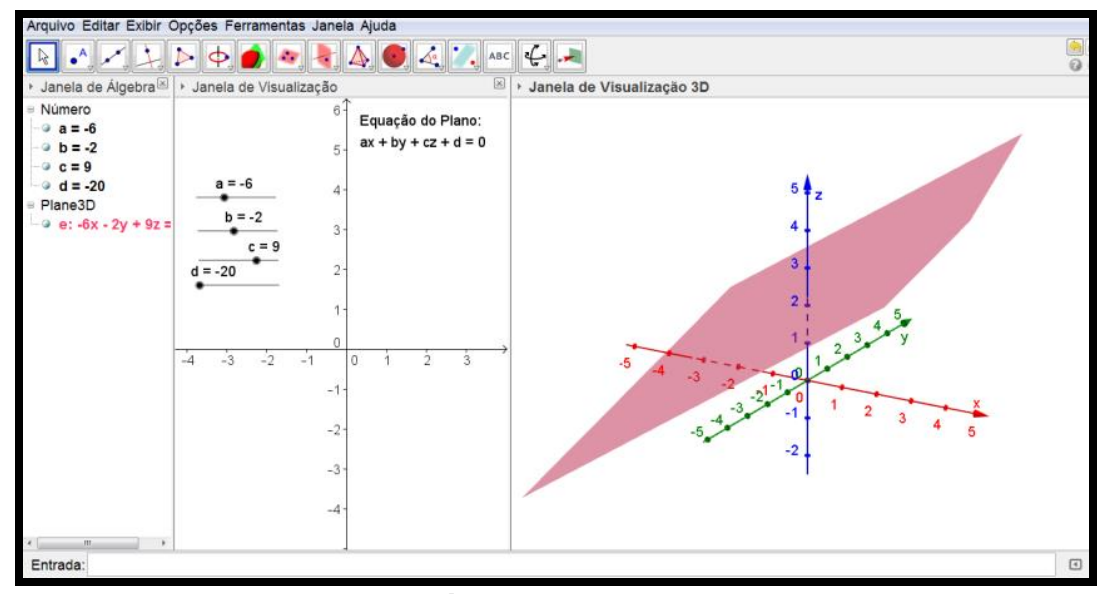

Figura 2. Applet

Fonte: <http://www.geogebratube.org/material/show/id/113179>

A apostila contém quatro atividades. A primeira é dividida em onze itens, nos quais a manipulação do applet é necessária para a resolução. A segunda contém sete itens, em cada um dos quais é apresentada a equação geral de um plano. A partir da análise dos coeficientes e do termo independente de cada equação, é solicitada a posição do plano em relação à origem, ou aos eixos coordenados, ou aos planos determinados por dois desses eixos, sem utilizar os recursos do applet. Ou seja, a resposta deve ser dada a partir das conjecturas estabelecidas na atividade 1 . As atividades 3 e 4 têm por objetivo generalizar os casos particulares da equação geral do plano. A atividade 3 é 
composta de cinco itens nos quais são solicitados exemplos de equações, a partir de uma dada condição dos coeficientes, sem utilizar o applet. Na atividade 4, o uso do applet é facultativo, nesta são dadas condições dos coeficientes e do termo independente da equação geral do plano e questionado sobre as posições particulares do plano (Figura3).

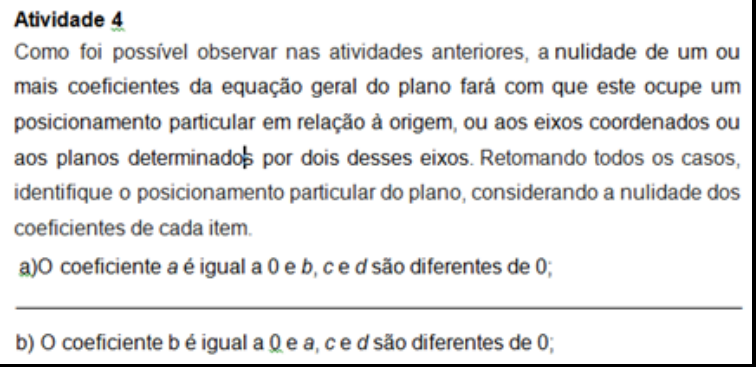

Figura 3. Parte da Atividade 4

Fonte: Elaboração própria.

A elaboração da apostila visou possibilitar o estudo do tema de forma dinâmica, favorecer a utilização do applet e contribuir para a visão de que o uso de TD na educação não pode ser desvinculado de um propósito pedagógico claramente definido. $\mathrm{Na}$ seção seguinte, analisam-se os dados levantados no estudo promovido.

\section{Análise e Discussão dos Dados}

Os licenciandos manifestaram muito interesse pela Janela 3D do GeoGebra 5.0 e testaram os recursos com facilidade. As orientações de uso das ferramentas contidas no próprio software foram fundamentais para a experimentação das mesmas. Ao apresentar a referida janela, visou-se divulgar um recurso novo, de grande potencial.

Somente dois alunos conheciam o GeoGebraTube, no entanto, todos fizeram o download do applet Equação Geral do Plano com facilidade, a partir dessa plataforma de compartilhamento.

Os licenciandos se mostraram muito interessados ao utilizar o applet e participaram ativamente da resolução das atividades. Os mesmos foram orientados a analisar os recursos durante a utilização destes. Quando as atividades foram concluídas, foi feita a correção oral, acompanhada de uma discussão. Por meio das respostas orais e da posterior análise das apostilas recolhidas, verificou-se que todos os alunos acertaram as atividades. Com relação ao applet, os participantes se mostraram bastante motivados e o utilizaram sem dificuldades. Oralmente, alguns destacaram a importância desse recurso para a análise dos casos particulares da equação geral do plano. Mas, além disso, sugeriram retirar do applet a equação do plano, pois a mesma estava atrapalhando a visualização, durante a movimentação. Em relação à apostila de atividades, alguns participantes propuseram, oralmente, mudanças na ordem das mesmas.

Ao final do minicurso, todos os licenciandos responderam ao questionário mencionado na seção anterior. Os resultados são discutidos a seguir. Dos sete, seis eram do sexo feminino e a média de idade do grupo era de 21 anos, com desvio padrão de, aproximadamente, um.

Todos os participantes já conheciam a equação geral do plano e apenas um respondeu que não havia estudado os casos particulares da equação do plano antes do minicurso. Aos que já tinham estudado, foram apresentadas opções que permitiram identificar que quatro estudaram o tema apenas por meio de aula expositiva e os outros 
dois por meio de aula expositiva, material impresso e concreto. A opção "Com apoio de software, vídeo ou algum outro tipo de recurso digital" não foi assinalada, indicando, assim, que essa foi a primeira experiência de estudo do tema com auxílio de TD.

Com relação à utilização do applet Equação Geral do Plano para resolução das atividades pedagógicas, foram propostas três afirmativas diante das quais os licenciandos deveriam assinalar uma das opções: Discordo Completamente (DC), Discordo (D), Não Concordo Nem Discordo (NCND), Concordo (C) e Concordo Completamente (CC). A Tabela 1 mostra os resultados. Os percentuais apresentados na mesma indicam que o applet foi bem avaliado pelos participantes e os ajudou a investigar os casos particulares da equação geral do plano. Como defendido por Borba (2011), as possibilidades de experimentação propiciadas pelas TD podem contribuir para o estabelecimento de conjecturas.

Tabela 1. Avaliação do Applet Equação Geral do Plano

\begin{tabular}{ccccc}
\hline $\begin{array}{c}\text { DC } \\
\%\end{array}$ & $\begin{array}{c}\mathrm{D} \\
\%\end{array}$ & $\begin{array}{c}\text { NCND } \\
\%\end{array}$ & $\begin{array}{c}\mathrm{C} \\
\%\end{array}$ & $\begin{array}{c}\mathrm{CC} \\
\%\end{array}$ \\
\hline $\begin{array}{c}\text { Afirmativa 1: Utilizar os recursos do applet foi fácil. } \\
0\end{array}$ & 0 & 0 & 14,3 & 85,7 \\
\hline $\begin{array}{l}\text { Afirmativa 2: A manipulação do applet contribuiu para o estabelecimento das conjecturas } \\
\text { solicitadas nas atividades. }\end{array}$ & \multicolumn{5}{l}{} \\
\hline 0 & 0 & 0 & 0 & 100 \\
\hline \multicolumn{5}{l}{ Afirmativa 3: A visualização dos planos foi satisfatória. } \\
\hline 0 & 0 & 0 & 28,6 & 71,4 \\
\hline
\end{tabular}

\section{Fonte: Elaboração própria.}

A próxima questão era aberta e solicitava comentários sobre o applet. Alguns dos registros feitos destacaram a importância do uso desse recurso, mas sugestões de melhorias também foram apresentadas, de forma semelhante ao que ocorreu durante a manipulação do applet. Além da não exibição da equação do plano, foi sugerida a diminuição da fonte do applet. Uma recomendação de uso inicial com alunos também foi mencionada: explicar que, quando o eixo se encontra tracejado, o mesmo não pertence ao plano exibido. Estes aspectos sinalizam que os licenciandos foram críticos na análise, o que se considera imprescindível para o uso das TD, como mencionam Lima et al. (2012), conforme discutido na seção 2 deste artigo. Além disso, esses dados foram importantes para as desenvolvedoras do applet (autoras deste artigo), pois mostraram uma boa aceitação do mesmo, mas também apontaram sugestões de melhorias. Como afirma Tarouco (2004), os feedbacks fornecidos por avaliações são importantes para os desenvolvedores a fim de que os recursos possam ser aprimorados.

Com relação às atividades, foram propostas quatro afirmativas diante das quais os licenciandos também deveriam assinalar uma das opções: Discordo Completamente (DC), Discordo (D), Não Concordo Nem Discordo (NC ND), Concordo (C) e Concordo Completamente (CC). A Tabela 2 mostra os resultados.

$\mathrm{Na}$ referida tabela, é possível observar que a maioria dos licenciandos considerou as atividades interessantes e com enunciados claros. Todos concordaram completamente que as mesmas contribuíram para a aprendizagem do tema. A afirmativa que apresentou menor percentual na opção Concordo Completamente foi a relativa à quantidade de atividades propostas. Essa quantidade era justamente uma preocupação 
das pesquisadoras, mas as mesmas optaram por manter a proposta original e verificar o posicionamento crítico dos participantes. Esses resultados apontam para o fato dos licenciandos estarem atentos a aspectos pedagógicos, o que é considerado importante na perspectiva teórica TPACK [Mishra; Koehler 2006].

Tabela 2. Avaliação das atividades

\begin{tabular}{|c|c|c|c|c|}
\hline $\mathrm{DC}(\%)$ & $\mathrm{D}(\%)$ & $\operatorname{NCND}(\%)$ & $\mathrm{C}(\%)$ & $\mathrm{CC}(\%)$ \\
\hline \multicolumn{5}{|c|}{ Afirmativa 1 : $\mathrm{O}$ enunciado das atividades estão claros. } \\
\hline 0 & 0 & 0 & 14,3 & 85,7 \\
\hline \multicolumn{5}{|c|}{ Afirmativa 2: As atividades são interessantes. } \\
\hline 0 & 0 & 14,3 & 14,3 & 71,4 \\
\hline \multicolumn{5}{|c|}{$\begin{array}{l}\text { Afirmativa 3: As atividades contribuíram para a construção de conhecimentos sobre o tema em } \\
\text { estudo. }\end{array}$} \\
\hline 0 & 0 & 0 & 0 & 100 \\
\hline \multicolumn{5}{|c|}{ Afirmativa 4: A quantidade de atividades proposta está adequada } \\
\hline 0 & 0 & 14,3 & 57,1 & 28,6 \\
\hline
\end{tabular}

\section{Fonte: Elaboração própria.}

Em uma questão aberta, que solicitava comentários sobre as atividades, os licenciandos registraram algumas sugestões, especificamente, em relação à atividade 4. Essa atividade tem por objetivo generalizar os casos particulares da equação geral do plano, mas a atividade 3 também já tinha esse objetivo, o que tornou cansativa a resolução. Nesse sentido, uma sugestão dada foi transformar a atividade $4 \mathrm{em}$ um quadro, para que não fique repetitiva, em relação à atividade 3 . Também foi sugerido que atividade 4 se tornasse a atividade 2, para que fosse promovida uma generalização imediatamente após a atividade 1. Tais propostas serão analisadas, tendo em vista identificar a melhor alteração a ser promovida na apostila. Essas sugestões sinalizam o envolvimento e a seriedade dos licenciandos no processo de avaliação dos recursos.

Questionados se utilizariam os applets e a apostila de atividades como futuros professores, todos os licenciandos responderam positivamente, apresentando justificativas relacionadas ao favorecimento da compreensão do tema.

Em relação ao uso de applets, de maneira geral, para abordagem de temas matemáticos, seis dos sete participantes avaliaram como Muito Bom e um (Licenciando 5) avaliou como Bom. Destacam-se três comentários sobre esse aspecto:

$\mathrm{O}$ uso do applet se faz necessário em diversos conteúdos matemáticos. $\mathrm{O}$ mesmo contribui para que o aluno visualize questões, por exemplo, que envolvam o 3D (Licenciando 2);

O applet foi um ótimo apoio para a explicação do conteúdo (Licenciando 5);

Sem dúvidas o uso de applets facilita a compreensão de um modo geral na explicação de conteúdos. Desenhos no quadro não tornam uma aula tão satisfatória quanto quando se usa tecnologia (Licenciando 7).

Estes comentários ratificam a visão de Borba (2011), quando o mesmo defende as potencialidades educacionais das TD.

As duas últimas questões solicitavam pontos positivos e negativos do minicurso. Nenhum licenciando enumerou pontos negativos e todos listaram positivos. Destacamse dois desses: 
Mostra a importância do uso da tecnologia em sala de aula, facilitador na construção do conhecimento, atrativo e interessante (Licenciando 4);

A atividade e a sequência utilizada foram muito interessantes, o debate feito durante a sequência didática foi algo enriquecedor para o nosso conhecimento, o uso do GeoGebra 3D foi surpreendente e motivador (Licenciando 6).

Esses comentários sinalizam que os licenciandos consideraram o minicurso importante e que o uso do GeoGebra 5.0, do applet e das atividades contribuíram para o estudo dos casos particulares da equação geral do plano. Além disso, ressaltam a importância de propor, na formação de professores, a análise de materiais pedagógicos, contribuindo para o desenvolvimento de atitudes críticas.

\section{Considerações Finais}

De maneira geral, o applet e as atividades foram avaliados positivamente pelos licenciandos. As sugestões recebidas serão analisadas e, a partir disso, melhorias serão promovidas nos recursos elaborados. É importante destacar que a versão 5.0 do GeoGebra, que foi a utilizada na elaboração do applet, é nova e ainda está passando por uma fase de testes. Porém, percebe-se que esse é um software com inúmeras possibilidades educacionais e que tem evoluído bastante. A proposta pedagógica associada ao GeoGebra 5.0 requer incentivo a investigações, a descobertas e a reflexões.

Além da importância para o estudo do tema em si, ações como a descrita contribuem para o desenvolvimento do senso crítico dos licenciandos e, até mesmo, para maior familiarização com procedimentos de pesquisa. Além disso, destaca-se a importância de trabalhar as TD dentro do contexto pedagógico do professor em formação, pois, assim, possibilita-se melhor compreensão de como essas tecnologias podem apoiar atividades pedagógicas. Tal proposta está adequada com o referencial TPACK.

Como estudos futuros, outros recursos digitais e atividades serão avaliados por licenciandos. Também se pretende propor minicursos nos quais licenciandos selecionem recursos e elaborem atividades. Finalizando, espera-se, com essas ações, estar contribuindo para uma formação de professores de Matemática mais adequada às características do século XXI, buscando assim, colaborar com o processo de ensino e aprendizagem de temas matemáticos.

\section{Referências}

Batista, S. C. F. (2004). SoftMat: Um Repositório de Softwares para Matemática do Ensino Médio - Um Instrumento em Prol de Posturas mais Conscientes na Seleção de Softwares Educacionais. Dissertação (Mestrado em Ciências de Engenharia) Universidade Estadual do Norte Fluminense - UENF, Campos dos Goytacazes, RJ.

Borba, M. C. (2011). Educação matemática a distância online: balanço e perspectivas, In: CONFERÊNCIA INTERAMERICANA DE EDUCAÇÃO MATEMÁTICA (CIAEM), 13, 2011, Recife, PE. Anais... Recife, PE, p. 1-9.

Borges, M. de F. V. (2008). Inserção da Informática no Ambiente Escolar: Inclusão Digital e Laboratórios de Informática numa Rede Municipal de Ensino. In: CONGRESSO SBC, XXVIII. WORKSHOP SOBRE INFORMÁTICA NA ESCOLA (WIE), 2008, Belém do Pará, PA. Anais... Belém do Pará, PA, p. 146-155. Disponível 
em: <http://www.br-ie.org/pub/index.php/wie/article/view/972>. Acesso em: 10 de jul. 2014.

Deitel, H. M; Deitel, P. J. (2003). Java, como programar. Tradução de Carlos Arthur Lang Lisboa. 4. ed. Porto Alegre: Bookman.

Drijvers, P. (2012). Digital technology in mathematics education: why it works (or doesn't). In: INTERNATIONAL CONGRESS ON MATHEMATICAL EDUCATION, 12, 2012, Seoul, Korea. Proceedings ...Seoul, Korea, July 8-15. Disponível em: <http://www.icme12.org/upload/submission/2017_F.pdf>. Acesso em: 10 mar. 2014.

Guedes, F. D.; Castro-Filho, J. A. A (2010). Seleção de objetos educacionais digitais por professores. In: SIMPÓSIO BRASILEIRO INFORMÁTICA DE INFORMÁTICA NA EDUCAÇÃO, XXI, 2010, João Pessoa, PA. Anais... João Pessoa, PA, p. 1-9. Disponível em: <http://www.br-ie.org/pub/index.php/sbie/article/view/1491/1256>. Acesso em: 10 jul. 2014

Hohenwarter, M.; Preiner, J. (2007). Dynamic mathematics with GeoGebra. The Journal of Online Mathematics and its Applications, v.7. Disponível em: <http://www.maa.org/external_archive/joma/Volume7/Hohenwarter/index.html>. Acesso em: 17 maio 2014.

Hohenwarter, M. (2013) GeoGebra 4.4 - from Desktops to Tablets. Indagatio Didactica, v. 5, n. 1, p. 8-18.

Klopfer, E.; Osterweil, O.; Groff, J.; Haas, J. (2009). Using the technology of today, in the classroom today: the instructional power of digital games, social networking, and simulations, and how teachers can leverage them. Disponível em: <http://education.mit.edu/papers/GamesSimsSocNets_EdArcade.pdf>. Acesso em: 11 mar. 2014.

Lima, L. de; Ribeiro, J. W.; Costa, M. J. N.; Loureiro, C. (2012). Reflexões sobre o Uso da Tecnologia Digital da Informação e Comunicação na Formação do Licenciando de Ciências. In: WORKSHOP SOBRE INFORMÁTICA NA ESCOLA (WIE), XVIII, 2012, Rio de Janeiro, RJ. Anais... Rio de Janeiro, RJ, p.1-10. Disponível em: <http://www.br-ie.org/pub/index.php/wie/article/view/2119> Acesso em: 10 jul. 2014.

Mishra, P.; Koehler, M. J. (2006). Technological Pedagogical Content Knowledge: a framework for teacher knowledge. Teachers College Record, v. 108, n. 6, June 2006, p. 1017-1054.

Nascimento, A. C. T. A. de A. (2013). Integração das Tecnologias às Práticas Escolares. In: Pesquisa sobre o uso das tecnologias de informação e comunicação no Brasil: TIC Educação 2012. São Paulo: Comitê Gestor da Internet no Brasil, 2013, p.45-49.

Tarouco, L. (2004). Avaliação de Objetos de Aprendizagem. Disponível em: <http://penta2.ufrgs.br/edu/objetosaprendizagem/sld001.htm>. Acesso em: 10 jun. 2014.

Zotto, N. D.; Machado, G. M. Z.; Mello, K. B. de; Silva, R. S. (2013). Geogebra 3D e quadro interativo: uma possibilidade para o ensino de geometria espacial no ensino médio. In: CONGRESSO INTERNACIONAL DE ENSINO DE MATEMÁTICA, 6, Canoas. Anais...Canoas: Universidade Luterana do Brasil, 16 - 18 de outubro, Canoas, 2013. p. 1-9. 